\title{
Reactive hydroxyapatite fillers for pectin biocomposites
}

Fabiola Munarin ${ }^{\mathrm{a}}$, Paola Petrinia*, Giulia Barcellona ${ }^{\mathrm{a}}$, Tommaso Roversi ${ }^{\mathrm{b}}$, Laura Piazza ${ }^{\mathrm{b}}$, Livia Visai ${ }^{\mathrm{c}, \mathrm{d}}$, Maria Cristina Tanzi ${ }^{\mathrm{a}}$

a Laboratorio di Biomateriali, Dipartimento di Chimica, Materiali e Ingegneria Chimica 'G. Natta' and UdR INSTM Milano Politecnico, Politecnico di Milano, Piazza Leonardo da Vinci 32, 20133 Milano, Italy

b DeFENS, Dipartimento per gli Alimenti, la Nutrizione e l'Ambiente, Università di Milano, 20133 Milano, Italy.

${ }^{\mathrm{c}}$ Dept. of Molecular Medicine, Center for Tissue Engineering (C.I.T.), INSTM UdR of Pavia, University of Pavia, 27100 Pavia, Italy

${ }^{\mathrm{d}}$ Dept. of Occupational Medicine, Ergonomy and Disability, Lab. Nanothecnology, Salvatore Maugeri Foundation, IRCCS, 27100 Pavia, Italy

* Corresponding author:

Paola Petrini, Politecnico di Milano, Piazza Leonardo da Vinci 32, 20133 Milano, Italy.

Tel. +39 0223993386, Fax +39 0223993360, Email: paola.petrini@polimi.it

\section{Abstract}

In this work, a novel injectable biocomposite hydrogel is produced by internal gelation, using pectin as organic matrix and hydroxyapatite either as crosslinking agent and inorganic reinforcement. Tunable gelling kinetics and rheological properties are obtained varying the hydrogels composition, with the final aim of developing systems for cell immobilization. The reversibility by dissolution of pectin-hydroxyapatite hydrogels is achieved with saline solutions, to possibly accelerate the release of the cells or active agents immobilized. Texture analysis confirms the possibility of extruding the biocomposites from needles with diameters from $20 \mathrm{G}$ to $30 \mathrm{G}$, indicating that they can be implanted with minimally - invasive approaches, minimizing the pain during injection and the side effects of the open surgery.

L929 fibroblasts entrapped in the hydrogels survive to the immobilization procedure and exhibit high cell viability. On the overall, these systems result to be suitable supports for the immobilization of cells for tissue regeneration applications.

Keywords: pectin; hydroxyapatite; injectable; hydrogels; biomaterials; internal gelation 


\section{Introduction}

Biocomposites, composite materials produced by natural sources, are commonly formed by an organic phase, prevalently polymeric, and an inorganic reinforcement [1,2]. Bone itself is an example of biocomposite material, being composed of collagen, the organic polymeric phase, which is intercalated by the inorganic hydroxyapatite phase $[3,4]$.

The aim of this work is focused on the formulation of novel injectable biocomposite hydrogels for minimally-invasive surgery, following an internal gelation method and employing the polysaccharide pectin as organic matrix and hydroxyapatite as inorganic phase. In the last decade, polysaccharide-based hydrogels have attracted more and more attention for biomedical applications, due to their high tissue-like water content, good biocompatibility and easy manufacturing [5-10]. These natural hydrogels are particularly suitable as injectable systems for regenerative medicine and tissue augmentation, due to the possibility of safely immobilizing stem cells in the hydrogel network, and to deliver them to the pathological site. However, some of the major goals of cell immobilization still need to be addressed, such as the cell migration outside of the hydrogel network and the formation of a new extracellular matrix in the damaged tissue, to accelerate the physiological healing process.

Injectable gels can be implanted by mini-invasive surgical procedures, thus requiring shorter preparation time and causing less morbidity than in the open surgery approach. These materials may cover a wide range of mechanical properties that can be tailored to the site of injection and therefore to their final application [5-10]. The number of commercially available injectable gels has increased significantly in the last years and recent efforts have been focused on the elimination of the deleterious foreign body and inflammatory reactions caused by some of the early injectable materials $[11,12]$.

Pectin is a natural polymer, easily available, mainly produced from citrus fruits and apples peel that has been recently exploited to reproduce the extracellular matrix for cell housing and tissue regeneration [13-17]. 
Low methoxyl (LM) pectins, e.g. pectins characterized by a degree of esterification (DE) lower than $50 \%$, form polyelectrolyte complexes in the presence of divalent ions, such as $\mathrm{Ca}^{2+}$ [8-23]. The gelling mechanism involves a chain-chain association of galacturonans and the creation of junction zones via calcium ions complexation, thus resulting in the formation of a physical gel. The mechanism of LM pectin gelation can be explained as a cooperative twostage process involving an initial dimerization, by the ionic complexation of the carboxyl groups of pectin chains through $\mathrm{Ca}^{2+}$ ions, followed by subsequent aggregation of the dimers [23]. The initial dimer associations are constituted by electrostatic interactions, efficiently stabilized by intermolecular hydrogen bonding and van der Waals forces. The second stage instead is characterized by weaker dimers associations, mainly governed by electrostatic interactions, and easily disrupted by counterions, such as $\mathrm{Na}^{+}$ions [23].

Pectin external gelation, promoted by the use of water-soluble calcium sources, such as $\mathrm{CaCl}_{2}$, gave interesting results for the formation of microspheres for cell immobilization [1315]. However, an internal gelation mechanism, employing poorly water-soluble $\mathrm{Ca}^{2+}$ sources, may improve the homogeneity of the hydrogels and provide tunable gelling kinetics. Hydroxyapatite was selected exploiting its scarce water solubility to obtain a double effect: pectin cross-linking with $\mathrm{Ca}^{2+}$ ions from the dissolved hydroxyapatite and inorganic reinforcement from the un-dissolved fraction, thus acting as reactive filler. Furthermore, combining the inorganic filler with the polymeric matrix can be used as a strategy to increase the mechanical properties of the injectable biocomposites.

Though polyelectrolyte complexes and pectin- based hydrogels have been reported several times in the literature [5-17], as far as we know, the production of pectin biocomposites by internal gelation using hydroxyapatite as source of calcium ions has never been exploited, and these systems hold great potentialities for tissue engineering applications. 


\section{Experimental Section}

\subsection{Materials}

Low methoxyl (LM) Pectin (Pectin classic CU 701, batch 01001018, DE=42\%, Mw= $261 \mathrm{kDa}$ and $\mathrm{Mn}=92 \mathrm{kDa}$ measured by intrinsic viscosity and GPC analysis, respectively ${ }^{24}$ ), was kindly provided by Herbstreith \& Fox (Neuebuerg, Germany). Calcium hydroxyapatite powder (granulometry: $d<40 \mathrm{~m}$, batch 79), custom- produced and provided by Eurocoating S.p.A (Pergine, Trento, Italy) was used for the preparation of the injectable gels.

Other chemicals, such as sodium hydroxide, ultrapure water, ethanol and sodium chloride, were supplied by Sigma Aldrich and used as received.

\subsection{Methods}

\subsubsection{Gel preparation}

Pectin and hydroxyapatite gels, named PectHA, were produced according to the procedure developed by the authors and described in an international patent [25]. Various hydrogels formulations were prepared with increasing concentrations of pectin (from $3 \% \mathrm{w} / \mathrm{v}$ to $7 \% \mathrm{w} / \mathrm{v}$ ) and hydroxyapatite (from $0.3 \%$ to $0.5 \%$ ).

Briefly, a $6 \%(\mathrm{w} / \mathrm{v})$ pectin solution (the $\mathrm{pH}$ adjusted to reach a final $0.05 \mathrm{mM} \mathrm{NaOH}$ in the hydrogels) was diluted with the appropriate amount of hydroxyapatite in water suspension. Soon after preparation, the mixtures were poured into $2 \mathrm{ml}$ glass syringes and were left at room temperature for $24 \mathrm{~h}$ to stabilize the gel networks.

After preparation, gels were kept at room temperature in the glass syringes and extruded with $30 \mathrm{G}$ needles before any characterization (unless otherwise specified).

$0.5 \mathrm{~g}$ of the hydrogel samples were extruded into Petri dishes $24 \mathrm{~h}$ after preparation and their $\mathrm{pH}$ was measured with a Eutech pH meter (EuTech Instruments, Europe B.V., 
Nijkerk, Netherlands) with a double pore slim electrode (Hamilton Bonaduz AG, Switzerland), appositely designed for soft gels and food measurements.

\subsubsection{Rheological properties}

Time sweeps, oscillation measurements and temperature ramp were performed with an AR-1500ex Rheometer (TA instrument, USA) using a parallel plate geometry $(20 \mathrm{~mm}$ diameter, $1.2 \mathrm{~mm}$ gap) to evaluate the rheological properties of the gels. All the rheological measurements were performed within the linear viscoelastic region. According to previous rheological studies of biological soft tissues, such as brain or adipose tissue [26-28], and injectable hydrogels [29-34], the following testing procedures and parameters were chosen.

To determine the gel point, i.e. the liquid-to-solid transition in soft materials [35], time sweeps were assessed in oscillatory mode at constant shear frequency $(\omega=0.7 \mathrm{~Hz})$, stress amplitude $(\sigma=5.0 \mathrm{~Pa})$ and temperature $\left(\mathrm{T}=25^{\circ} \mathrm{C}\right)$ for 60 minutes.

Frequency sweep measurements were performed at $\sigma=5 \mathrm{~Pa}$ over a frequency range of $0.1-10 \mathrm{~Hz}$, at temperatures of $25^{\circ} \mathrm{C}$ and $37^{\circ} \mathrm{C}$. This narrow range of frequency refers to the physiological stresses to which soft tissues are commonly subjected [30-33]. Storage modulus (G') and complex viscosity $\left(\eta^{*}\right)$ values were extrapolated at the frequency of $0.7 \mathrm{~Hz}$, which is typically used to compare the rheological properties of injectable hydrogels [31-33]. To determine the aging dynamic, the results of frequency sweep tests for a $3 \%(\mathrm{w} / \mathrm{v})$ pectin solution gelled in presence of $0.5 \%(\mathrm{w} / \mathrm{v})$ hydroxyapatite were considered for a period of time ranging between 35 minutes, roughly corresponding to the gel point, and 180 minutes.

Temperature sweep studies were assessed to determine the variation of complex viscosity with temperature at a fixed frequency $(\mathrm{f}=0.1 \mathrm{~Hz})$. The experiments were carried out by heating the samples with a temperature ramp of $2.5^{\circ} \mathrm{C} / \mathrm{min}$ from $20^{\circ} \mathrm{C}$ to 
$60^{\circ} \mathrm{C}$. This temperature range includes either the physiological temperatures $\left(36^{\circ} \mathrm{C}-\right.$ $42^{\circ} \mathrm{C}$ ) either a simulation of the environmental temperatures that the hydrogels might experience in stock conditions.

\subsubsection{Determination of injectability}

The injection forces needed to extrude the injectable PectHA hydrogels from syringes with different needles (with internal diameters of $25 \mathrm{G}$ and $30 \mathrm{G}$ ) or without a needle were evaluated with a texture analyser (TA.XT plus, Texture Technologies). The glass syringes containing $2 \mathrm{ml}$ of the biocomposite hydrogels were placed in the texture analyser and exposed to a constant injection speed of $100 \mathrm{~mm} / \mathrm{min}$ at $30 \mathrm{~mm}$ displacement (extruding approximately the $90 \%$ of the gel volume). The average force required to inject the gel was calculated in a distance range of 10 to $20 \mathrm{~mm}$. Samples were tested in triplicates and the results are expressed as the average injection force, with the standard deviations indicating the error.

\subsubsection{In vitro dissolution}

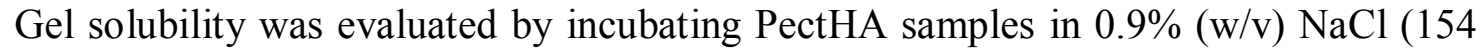
$\mathrm{mM}$ ) solutions as dissolving medium. The protocol assessed in this work consisted in injecting $0.1 \mathrm{~g}$ of the biocomposite hydrogel in a glass vial containing $0.5 \mathrm{ml}$ of $0.9 \%$ $(\mathrm{w} / \mathrm{v}) \mathrm{NaCl}$. The gels were immersed in the $\mathrm{NaCl}$ solution, then, at each time stage $(0$, 10,20 and 60 minutes), the dissolved gel was aspirated with a syringe, through a $18 \mathrm{G}$ needle, and the remaining gel was weighted in its wet form. The weight loss was calculated with the following equation (Equation 1):

$$
\Delta W=\left[\left(w_{t}-w_{0}\right) / w_{0}\right] \times 100
$$

where $\mathrm{w}_{\mathrm{t}}$ is the wet weight of the extruded gel at time point $\mathrm{t}$ and $\mathrm{w}_{0}$ is the wet weight at time 0 . 


\subsubsection{Cell culture}

L929 murine fibroblasts (American Type Culture Collection, Rockville, MD) were cultured in RPMI-1640 medium (Gibco) supplemented with 10\% fetal bovine serum, $1 \%$ L-glutamine, $1 \%$ sodium pyruvate, and 1\% antibiotics (Sigma-Aldrich, St. Louis, MO, USA). The cells were incubated at $37{ }^{\circ} \mathrm{C}$ with $5 \% \mathrm{CO}_{2}$, routinely trypsinized after confluency, counted, and seeded on 96 multi-well culture plates.

\subsubsection{Indirect cytocompatibility}

The indirect cytocompatibility assay was performed on PectHA samples according to ISO10993-5 standard practice. Briefly, $0.4 \mathrm{~g}$ of the samples, appositely prepared in sterile conditions, were extruded from the syringe and incubated in $2 \mathrm{ml}$ of ultrapure water (Sigma). After $24 \mathrm{~h}$ of incubation, the extracts were collected and tested with the MTT assay.

L929 cells were seeded at a density of $10^{4}$ cells/well in a 96-well culture plate. After 12 $\mathrm{h}$ of incubation, the culture medium was removed and $40 \quad 1$ of the extract with $160 \mu \mathrm{l}$ of fresh culture medium were added in each well. The negative control consisted in 40 1 of ultrapure water instead of the extract.

Cells where then incubated at $37^{\circ} \mathrm{C}$ in a $5 \% \quad \mathrm{CO}_{2}$ atmosphere, and 3-(4,5dimethylthiazole-2-yl)-2,5-diphenyl tetrazolium bromide (MTT) assay was performed after $48 \mathrm{~h}$ of incubation. The sample was removed and replaced by $100 \mathrm{~L}$ of fresh culture medium without serum. 10 L of MTT solution $(5 \mathrm{mg} / \mathrm{mL}$ in phosphatebuffered saline) was added to each well and the cell cultures were incubated for $3 \mathrm{~h}$. After removing the MTT solution, 100 L of acid isopropanol (Sigma Aldrich) were added to solubilize the formazan salts produced by viable cells. Aliquots of $200 \mathrm{~L}$ were sampled, and the absorbance was measured at $595 \mathrm{~nm}$ by a Biorad iMark microplate reader (BioRad Laboratories, Hercules, CA) [24]. 
All measurements regarding cell viability were tested in triplicate. ANOVA test was used to compare the data, and the results were considered statistically different when $p$ $<0.05$.

\subsubsection{L929 cell immobilization}

To evaluate the potentiality of pectin-hydroxyapatite hydrogels for regenerative medicine, cell immobilization was performed using L929 fibroblasts. Briefly, the fibroblasts $\left(\right.$ cell density $=8 \times 10^{5}$ cells $\left./ \mathrm{ml}\right)$ were suspended in a $6 \%(\mathrm{w} / \mathrm{v})$ pectin solution prepared in $0.9 \%(\mathrm{w} / \mathrm{v}) \mathrm{NaCl}$. The cell-polymer suspension was cross-linked with $0.5 \%$ $(\mathrm{w} / \mathrm{v})$ hydroxyapatite water suspension, to gain a final pectin concentration of $3 \%$ $(\mathrm{w} / \mathrm{v}) .0 .5 \mathrm{~mL}$ of the gelling mixture was then put in each well of a 48 -wells culture plate. After gelation, $1 \mathrm{ml}$ of culture medium was added in each well and the plates were incubated in controlled atmosphere $\left(\mathrm{T}=37^{\circ} \mathrm{C}, 5 \% \mathrm{CO}_{2}\right)$.

To evaluate cell viability, Trypan Blue assay was performed after $1 \mathrm{~h}, 2 \mathrm{~h}, 4 \mathrm{~h}$ and $24 \mathrm{~h}$ of incubation, as follows. Cell pellets were recovered after dissolving the hydrogels with 1 $\mathrm{ml}$ of $0.9 \%(\mathrm{w} / \mathrm{v}) \mathrm{NaCl}$ added with $5 \mathrm{mM}$ EDTA and centrifuging the samples at 1300 rpmi for 3 minutes. Then, the pellet was re-suspended with 80 L of Trypan blue solution and 20 L of fresh culture medium. $6.6 \mathrm{~L}$ of the sample was drawn into KOVA Slide2 chamber (Glasstic Slide 10, KOVA International) and the cells were counted at the optical microscope (Olympus IX50). Cell viability (\%) was expressed as (live cell number/ number of immobilized cells)*100.

\section{Results}

The internal gelation of pectin with hydroxyapatite resulted in the production of homogeneous soft hydrogels. Their tunable gelling kinetics allowed to load the hydrogels in disposable glass syringes, and possibly to inject them, prior to gelling. 
The initial $\mathrm{pH}$ of $6 \%(\mathrm{w} / \mathrm{v})$ pectin solution was $4.30 \pm 0.20$. When adding the crosslinker, i.e. hydroxyapatite, the $\mathrm{pH}$ slightly increased, until reaching $\mathrm{pH} 4.70 \pm 0.2$ for each formulation.

\subsection{Determination of the gel point}

Time sweep tests were assessed to evaluate the possibility to obtain in situ gelling systems or to manufacture gels that can be loaded in syringes during the gelling process. An example of the gel point is reported in Figure 1: during gelation, the sample changes from a liquid-to-solid like behaviour.

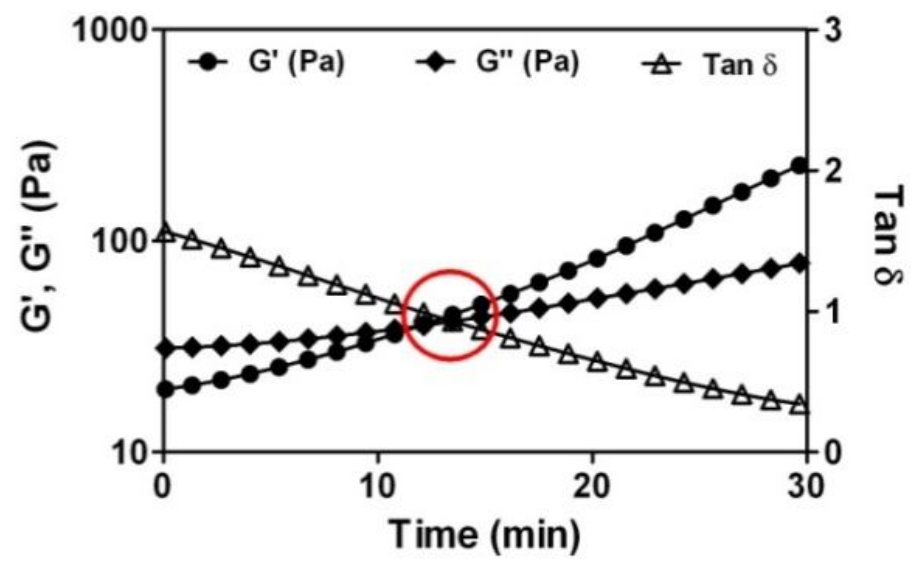

Figure 1. Storage modulus, loss modulus and tan $\delta$ of P5H03 gel. The gel point, indicated by the circle, occurs at 14 minutes, where the crossover of storage and loss moduli is reached and the value of tan $\delta$ is 1 .

Rheological measurements allow direct determination of the gel point, that is the time at which G' and G" cross each other $[16,35]$. In such an experiment, the evolution of G' and G' is measured in small amplitude oscillatory shear as a function of crosslinking time t. The frequency $\omega$ is kept constant throughout. At the beginning of the experiment, the value of G' is higher than G' and, after gelation, this order is reversed (Figure 1). Table 1 lists the experimental gelling time obtained for pectin hydrogels prepared by using different concentration of polymer and cross-linking agent. 
According to the collected data (Table 1), the use of higher amounts of hydroxyapatite resulted in faster gelling kinetics, while no clear trends were observed when pectin concentrations were varied up to $7 \%(\mathrm{w} / \mathrm{v})$.

Table 1. Gelling time of PectHA gels $\left(\omega=0.7 \mathrm{~Hz}, \mathrm{~T}=25^{\circ} \mathrm{C}\right)$.

\begin{tabular}{cccc}
\hline Formulation & $\begin{array}{c}\text { Pectin } \\
(\%)\end{array}$ & $\begin{array}{c}\text { Hydroxyapatite } \\
(\%)\end{array}$ & $\begin{array}{c}\text { Gelling } \\
\text { time } \\
(\text { min })\end{array}$ \\
\hline P3H0.3 & 3 & 0.3 & 48 \\
P3H0.5 & 3 & 0.5 & 35 \\
P5H0.3 & 5 & 0.3 & 14 \\
P5H0.5 & 5 & 0.5 & 9 \\
P7H0.3 & 7 & 0.3 & 33 \\
P7H0.5 & 7 & 0.5 & 19 \\
\hline
\end{tabular}

\subsection{Aging dynamic}

After reaching the gel point, the storage and loss moduli of pectin hydrogels were observed to increase up to $3 \mathrm{~h}$ after preparation. For longer times, the rheological properties were kept almost constant (Figure S1). Accordingly, the aging dynamic of the hydrogels was evaluated up to 3 hours. The double-log plot in Figure 2 shows the effect of frequency on the two dynamic moduli, G' and G'.

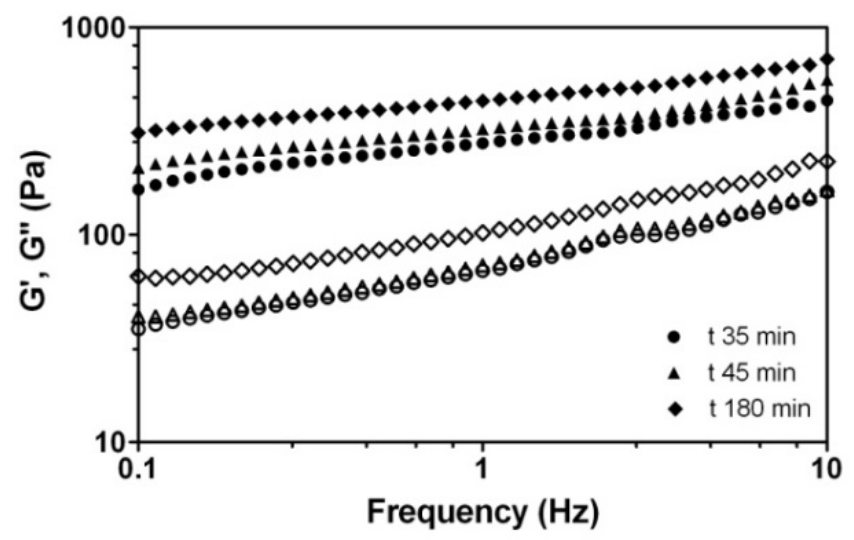

Figure 2. Storage ( $G$ ', filled symbols) and loss ( $G$ ', empty symbols) moduli of P3H05 biocomposite hydrogel as function of frequency and at different aging times: 35, 45 and $180 \mathrm{~min}$. 
For all samples G' exceeded G" by approximately a factor 5. A slightly linear behaviour was found for all the tested samples, thus indicating the possibility to calculate a realistic value of the coordination number as reported by Bohlin et al. [36]. Recalling basic theory, in a relaxation experiment a critical gel is characterized by a power law of the relaxation modulus (Equation 2)

$G(t)=A t^{n}$

where $A$ represents the gel strength and $\mathrm{n}$ is the relaxation exponent [37]. In our experiment we performed an oscillatory test at fixed frequency, $\omega$, that is qualitatively equivalent to a transient experiment at time $\mathrm{t}=1 / \omega$; thus, it may be assumed that Equation 2 transforms into Equation 3:

$G(\omega)=A \omega^{n}$

This critical gel is inherently assumed as the onset of a solid and therefore it relaxes in an infinite time and does not flow, the viscosity being infinite. In physical gelation, an important cooperative mechanism in pectin gelling, the stoichiometry of the physical cross-link is not easily controlled and the cross-link themselves may be labile with time under stress. Gels of this kind were referred as "weak" gels by de Gennes [38] since their cross-links have a binding energy $\sim \mathrm{kT}$ and so fluctuate in number and position, causing an actual fluctuation in the state of gelation. This process gives rise to a finite terminal relaxation time so that any applied strain can be eventually relaxed. To predict the suggested flow character, Mita et al. [39] proposed that the real structure of such a material is substituted by cooperative arrangement of flow units, to form a strand. Bohlin [36] demonstrated that in a relaxation experiment $G \sim \mathrm{t}^{-1 / z}$, where $\mathrm{z}$ is the coordination number. Applying this approach to physical gels, it can be suggested that the extent of interactions is measured by the interaction factor $\mathrm{z}$ that assumes the meaning of the number of rheological units correlated with one other in the three 
dimensional structure. Thus, in a dynamic oscillatory experiment $\mathrm{G}^{*}$ can be calculated as described in Equation 4

$G^{*}(w)=\sqrt{G^{\prime}(\omega)^{2}+G^{\prime \prime}(\omega)^{2}}=A_{f} \omega^{1 / z}$

Where $A_{f}$ is similar to $A$ of Equation 2 and 3, but refers to a flowing gel and may be interpreted as the strength of the interactions between those units [40]. The double log plot in Figure 3 (main body) shows as an example the effect of frequency on the complex modulus $\mathrm{G}^{*}$ for different aging times. For the sake of clarity, only three curves are here shown ( $\mathrm{t}=35-45-180$ minutes $)$.

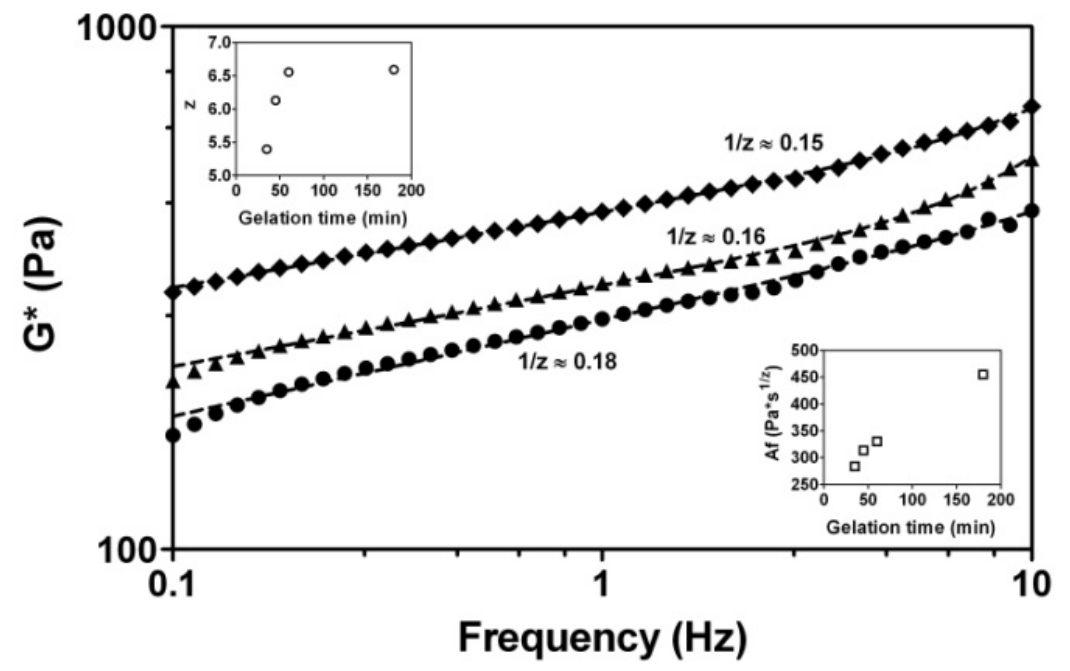

Figure 3. Evolution of $G^{*}$ with frequency at different aging times: $\bullet=35 \mathrm{~min}, \boldsymbol{\Delta}=45$ min and $=180 \mathrm{~min}$. In the insets, $z$ and Af parameters as calculated by fitting the experimental data with Equation 4.

As expected, $\mathrm{G}^{*}$ varies as a power law of frequency for all the tested samples (i.e. a straight line in the log-log representation). The upper left inset shows that the interaction parameter $\mathrm{z}$ calculated from the fitting of experimental data with Equation 4 grows roughly linearly with gelation time, increasing from $\approx 5.5$ at $\mathrm{t}=35 \mathrm{~min}$ to $\approx 6.5$ at $\mathrm{t}=60 \mathrm{~min}$. This linear dependence of $\mathrm{z}$ from time $(\mathrm{t})$ is limited however to aging times not exceeding 60 minutes, and is followed by a plateau value of the interaction parameter $\approx 6.5$ which is independent of sample aging up to 72 hours (data not shown). 
The latter observation, together with the fact that $A_{f}$ grows from $\approx 180 \mathrm{~Pa}^{*} \mathrm{~s}^{1 / \mathrm{z}}$ when $\mathrm{t}=$ $35 \mathrm{~min}$ to $\approx 450 \mathrm{~Pa}^{*} \mathrm{~s}^{1 / \mathrm{z}}$ at $\mathrm{t}=180 \mathrm{~min}$ (lower right inset of Figure 3 ), indicates that the number and the strength of the interactions is increasing with aging time. It is important to mention that the presence of these new links do not affect the overall viscoelastic properties of the gel network, being $1 / \tan \delta \approx 5$ for all the tested samples, as reported above.

\subsection{Rheological characterization of the extruded hydrogels}

To determine the rheological properties of the hydrogels after injection, frequency sweep tests were performed on pectin hydrogels extruded by $30 \mathrm{G}$ needles, $24 \mathrm{~h}$ after preparation.

A first clear evidence that can be extracted from the dynamic measurements shown in Figure 4 is that the magnitude of the storage modulus G' (Pa) and of the complex viscosity increase with pectin concentration and hydroxyapatite content.

A detailed analysis of the different curves shows that the frequency dependence of the elastic modulus remains the same regardless of polymer and gelling agent concentration, as it is clearly shown by a constant slope of $\approx 0.1$. Thus, all gels have a similar number of coordination. The shift of the storage modulus along yaxis towards higher values is following the increase of the solid content of the gels, resulting from pectin and un-dissolved hydroxyapatite. Considering that the $\mathrm{pH}$, and therefore the hydroxyapatite solubility, is similar for all of the produced hydrogels, the gel structure is retained, being dependent on the amount of free calcium ions available for the crosslinking.

Tan $\delta$ values were comparable for all the considered formulations, all of them resulting $<1$, thus indicating the solid-like behaviour of the gels [5]. 
A)

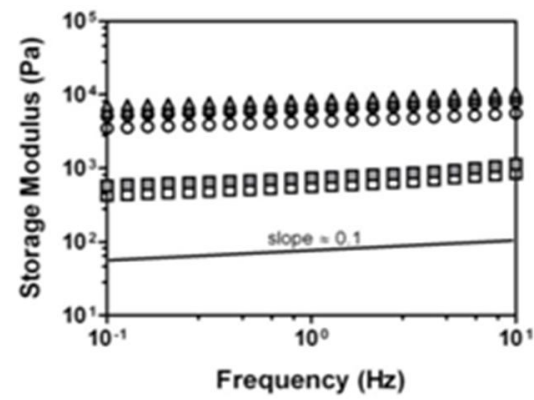

B)

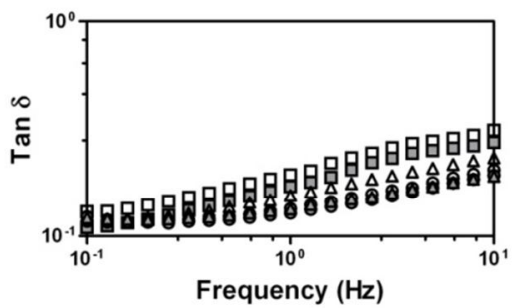

C)

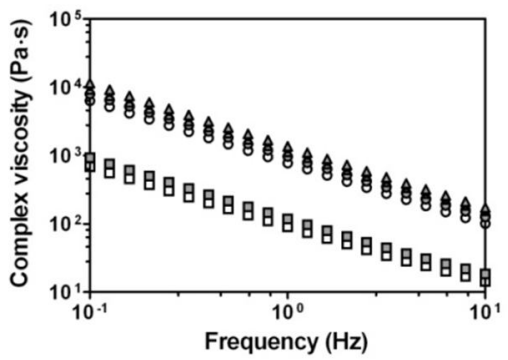

口 $\mathrm{P}_{3} \mathrm{H} 0.3$ ○ $\mathrm{P} 5 \mathrm{H} 0.3$ А $\mathrm{P} 7 \mathrm{H} 0.3$

a $\mathrm{P} 3 \mathrm{H} 0.5$ ○ $\mathrm{P} 5 \mathrm{H} 0.5$ \ $\mathrm{P} 7 \mathrm{H} 0.5$

Figure 4. Storage modulus (A), tan delta (B), and complex viscosity (C) of PectHA samples.

The rheological properties of each formulation were extrapolated at $\omega=0.7 \mathrm{~Hz}$ and $\mathrm{T}=$ $30^{\circ} \mathrm{C}$, as reported in Figure 5 and in Table 2.

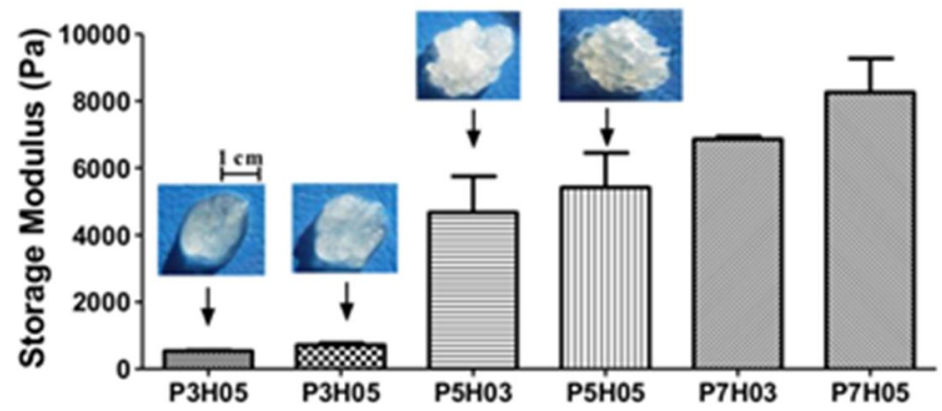

Figure 5. Storage modulus of PectHa gels extrapolated at $\omega=0.7 \mathrm{~Hz}, \mathrm{~T}=30^{\circ} \mathrm{C}$. Images of P3H03, P3H05, P5HO3 and P5HO5 extruded gels are reported to show the macroscopic texture of the hydrogels. Scale bar $=1 \mathrm{~cm}$. 
Table 2. Dynamic rheological properties of PectHa gels at $\omega=0.7 \mathrm{~Hz}$ and $\mathrm{T}=30^{\circ} \mathrm{C}$

\begin{tabular}{ccccc}
\hline Sample & $\boldsymbol{G}^{\prime}(\boldsymbol{P a})$ & $\boldsymbol{G}^{\prime \prime}(\boldsymbol{P a})$ & $\left|\boldsymbol{\eta}^{*}\right|(\boldsymbol{P a s})$ & Tan $\boldsymbol{\delta}$ \\
\hline P3H03 & 536 & 116 & 122 & 0.17 \\
P3H05 & 705 & 111 & 160 & 0.15 \\
\hline P5H03 & 4521 & 751 & 1024 & 0.12 \\
P5H05 & 6131 & 758 & 1389 & 0.12 \\
\hline P7H03 & 6825 & 993 & 1865 & 0.14 \\
P7H05 & 8227 & 1061 & 1550 & 0.12 \\
\hline
\end{tabular}

According to the results, hydrogels with tunable rheological properties were obtained. Further rheological characterization was performed to evaluate PectHa samples under temperature ramps (Figure 6).

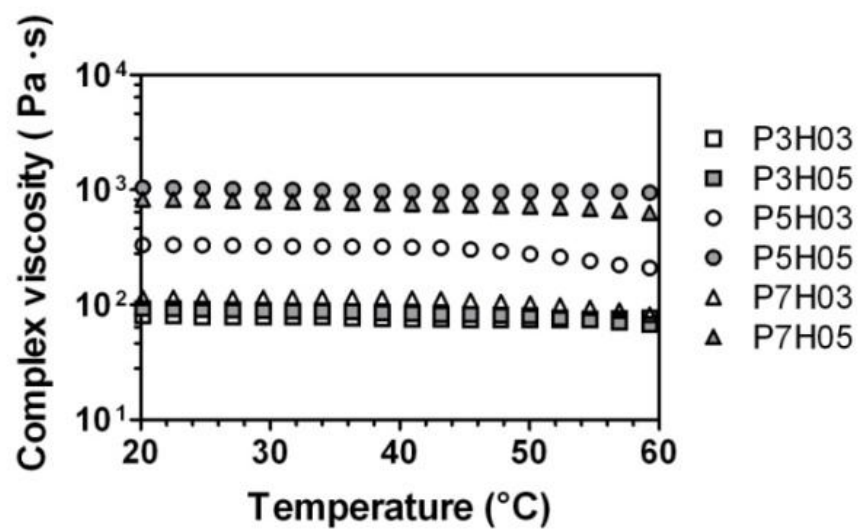

Figure 6. Temperature ramp test on PectHa hydrogels.

PectHa gels prepared with higher amounts of hydroxyapatite resulted stable over the whole range $\left(20^{\circ} \mathrm{C}-60^{\circ} \mathrm{C}\right)$ of temperature investigated (Figure 6). On the contrary, P3H03, P5H03 and P7H03 formulations showed a slightly decreasing trend of complex viscosity, associated to the weakening of the gel network at higher temperatures (Figure 6), probably due to the rupture of the secondary interactions, such as hydrogen bonds and van der Waals forces.

\subsection{Injectability test}

To measure the forces required to extrude the gels through a syringe, PectHa samples were tested with a texture analyzer. Gels were extruded from needles with different 
diameters (25G and $30 \mathrm{G})$ and without needle (the internal diameter of the tip of the syringe being $1.47 \mathrm{~mm}$ ). The injection force was increasing in the first seconds of the experiment, probably due to the extrusion of the air retained in the needle hub of the syringe, then it stabilized to a value that was kept constant until the end of the experiment (Figure 7A). In some cases, the injection force was oscillating up to the end of the test, probably due to a slight non-homogeneity of the biocomposites. These samples were not considered to calculate the mean injection force.

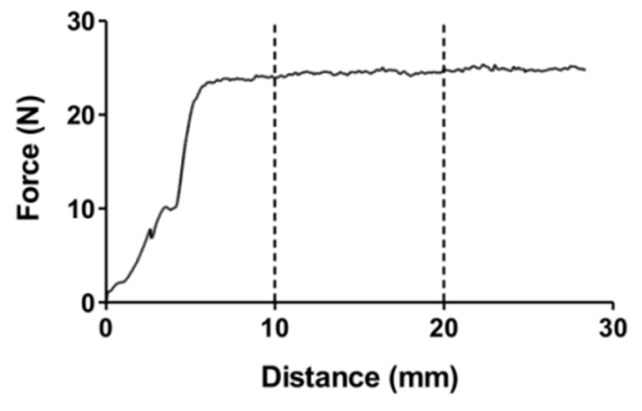

A)

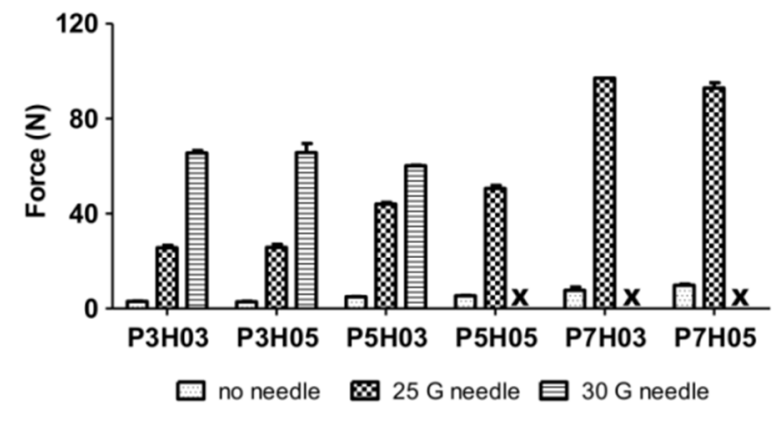

Figure 7. Plot of the injection forces required to extrude P3H05 by a $25 G$ needle syringe (A) and average injection forces of different needle sizes (B). The extrusion by $30 G$ needle syringes was not achieved for P5H05, P7H03 and P7H05 formulations ( $x$ symbols).

The injection force was calculated as the average force from $10 \mathrm{~mm}$ to $20 \mathrm{~mm}$ displacements (Figure 7A). The extrusion from different needles showed a remarkable increase of the injection force associated to the reduction of the needle diameter (Figure 7B). 
According to the results of the rheological analysis (Figure 4 and 5), the gels with lower pectin concentrations resulted softer and more flexible and therefore easier to extrude from small diameter needles.

\subsection{Hydrogel controlled reversibility}

The competing binding of monovalent ions, such as $\mathrm{Na}^{+}[21]$, can be exploited to trigger the complete dissolution of the hydrogels. This chararcteristic can provide a simple tool to remove the implanted hydrogels by inducing their dissolution.

Each formulation was dissolved after 30 minutes of static incubation in the dissolving medium $(0.9 \% \mathrm{w} / \mathrm{v} \mathrm{NaCl}$, Figure 8) and the resulting small gel fragments were collected with a $18 \mathrm{G}$ needle syringe.

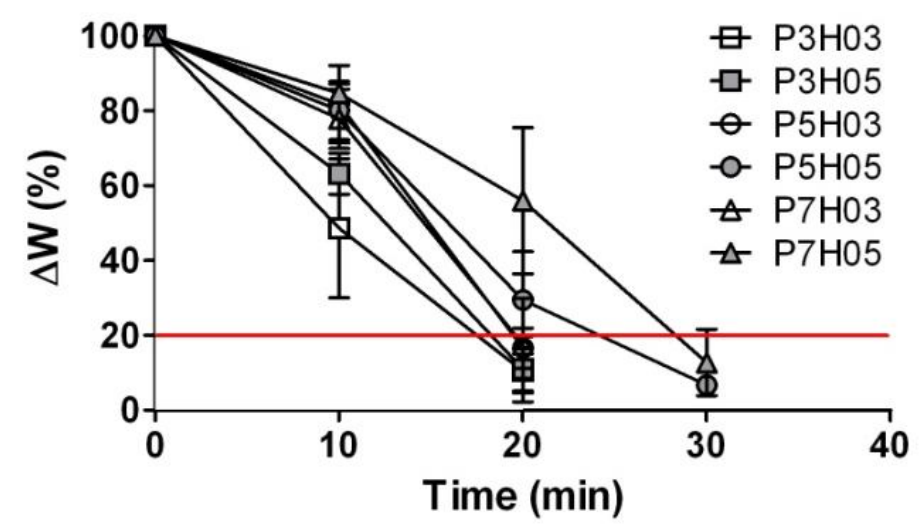

Figure 8. Dissolution test on PectHa samples. The complete dissolution was reached when the dissolved sample could be completely collected in a $18 G$ needle syringe, i.e. when the samples lost more than $80 \%$ of their initial weight (red line).

The dissolution rate resulted to be correlated to the hydrogel composition: the less entangled and less cross-linked hydrogels (P3H03, $\mathrm{P} 3 \mathrm{H} 05$ and $\mathrm{P} 5 \mathrm{H} 03)$, i.e. the hydrogels with lower amounts of pectin and hydroxyapatite, respectively, were dissolved faster than the stronger formulations (such as $\mathrm{P} 5 \mathrm{H} 05, \mathrm{P} 7 \mathrm{H} 03$ and $\mathrm{P} 7 \mathrm{H} 05$ ). This effect can be explained according to the increased spacing among pectin chains 
and to the more deformable structure of the weakest hydrogels, which may lead to a faster diffusion of $\mathrm{Na}^{+}$ions in the gel structure.

\subsection{Stability in different media and indirect cytocompatibility}

Before performing the in vitro characterization with L929 cell lines, the stability of the produced hydrogels was evaluated in the culture medium and in other aqueous media buffered at different $\mathrm{pH}$, in the absence of $\mathrm{Na}^{+}$ions, to simulate either the gastric either the intestinal $\mathrm{pH}$. After a first swelling phase, the hydrogels maintained a constant weight (Figure S2).

The cytocompatibility of L929 cells exposed to the gel extracts resulted comparable or higher than with the viability of the control (L929 cells cultured without the gel extracts) for each analyzed formulation. The viability of cells cultured in the presence of the P5 extracts resulted significantly lower than the viability of P7 extracts, as indicated by the analysis of variance (ANOVA) in Figure 9.

On the overall, these experiments indicate that PectHA gels are cytocompatible towards the fibroblast model.

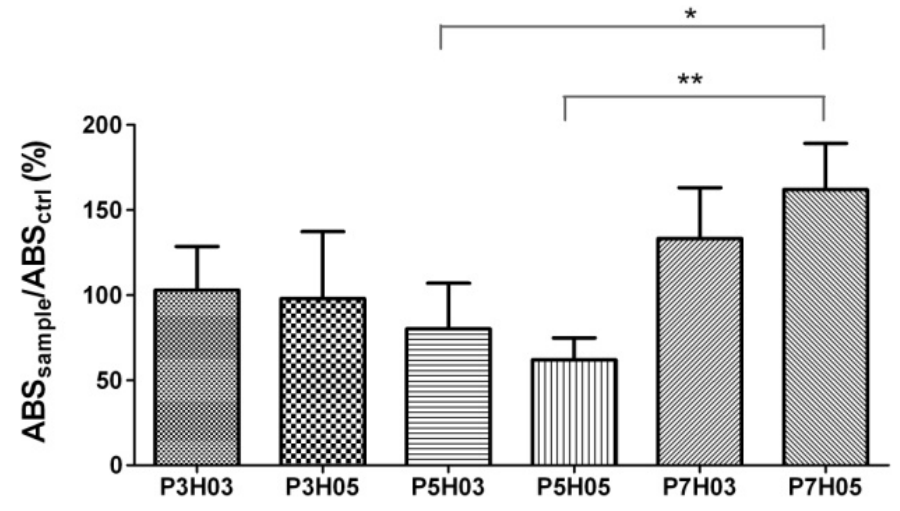

Figure 9. Indirect cytotoxicity of PectHA gels after $48 \mathrm{~h}$ of incubation. The reported values are related to the viability of L929 cells cultured without the gels extracts (100\%). A statistically significant difference was observed among P5H03 and P7H05 samples $(*, p<0.05)$ and among P5H05 and P7H05 samples $\left({ }^{* *}, p<0.01\right)$. 


\subsection{L929 cell immobilization}

Figure 10 reports the results of the relative cell viability of L929 fibroblasts immobilized in $\mathrm{P} 3 \mathrm{H} 05$ biocomposite hydrogels. As shown in the results, cells survived to the immobilization procedure and kept a constant viability up to $24 \mathrm{~h}$, thus indicting that $\mathrm{P} 3 \mathrm{H} 05$ hydrogels can provide an ECM-like environment able to support cell viability in the short-term culture.

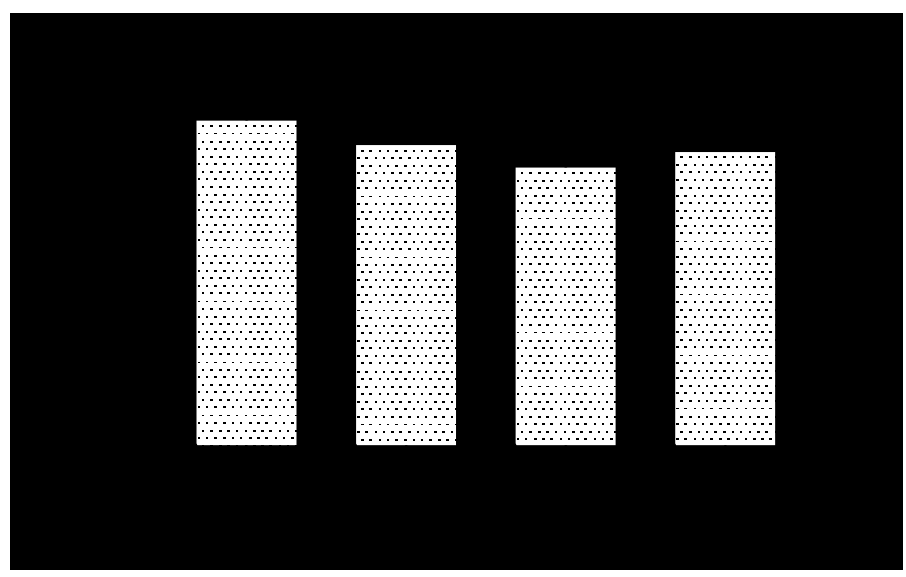

Figure 10. Relative cell viability of L929 fibroblasts immobilized in P3H05 hydrogels. The percentage of live cell is expressed taking as reference (100\%) the total number of immobilized cells.

\section{Discussion}

Natural polysaccharide-based hydrogels have been exploited in a wide range of biomedical and pharmaceutical applications. In the last decade, they are receiving increased attention as injectable gels for regenerative medicine [10, 41, 42]. The main aim of this work was to prepare and characterize an injectable biocomposite suitable for regenerative medicine applications, the matrix being pectin and the reinforcement hydroxyapatite. Though many examples of biocomposites produced with natural polysaccharides and hydroxyapatite are available in the literature [43-47], the amount of hydroxyapatite is usually predominant over that of the natural polymer, which is mainly employed as a plasticizer for the reinforcing particles. According to their mechanical 
strength, these biocomposites are mostly exploited for bone regeneration or as bone cements [43-48].

Our aim consisted in using the hydroxyapatite prevalently as a cross-linking agent of pectin, minimizing its role as reinforcement (Figure 11). Therefore, very low amounts of hydroxyapatite $(300 \mathrm{mg} / \mathrm{ml}$ to $500 \mathrm{mg} / \mathrm{ml})$ were used to produce the injectable biocomposites, but nonetheless obtaining injectable systems with different properties.

The mechanism of pectin gelling is commonly referred as internal gelation, and it is based on the slow dissolution of poorly soluble sources of mutivalent cations dispersed in pectin solutions [16]. As the $\mathrm{pH}$ of pectin is slightly acid $(\mathrm{pH}=4.30 \pm 0.20)$, hydroxyapatite can slowly dissolve releasing the $\mathrm{Ca}^{2+}$ ions that cross-link the carboxylate $\left(\mathrm{COO}^{-}\right)$groups of pectin chains. However, a certain amount of hydroxyapatite is not dissolved and remains in the polymeric matrix as reinforcement phase. The presence of un-dissolved hydroxyapatite, even at $0.3 \%(\mathrm{w} / \mathrm{v})$ concentration, indicates that $\mathrm{Ca}^{2+}$ is saturating pectin solutions, at the equilibrium, for all the formulations employed in this work. Thicker hydrogels are resulting from the higher amount of un-dissolved hydroxyapatite. The results of the gel point (Table 1) clearly indicate a faster gelling kinetic for the higher hydroxyapatite concentrations. It could be hypothesized that the larger surfaces available, the faster the dissolution is.

The polymeric hydrogels employed as injectable or implantable biomaterials should have a $\mathrm{pH}$ as near as possible to neutral in order to support cell viability and to avoid the necrosis of the surrounding tissues. Thus, the preparation of the pectin - hydroxyapatite biocomposite hydrogels was optimized with the addition of $\mathrm{NaOH}$ so to reach a suitable range of $\mathrm{pH}$ for each formulation.

The gelling kinetic of the injectable hydrogels was tuned to be sufficiently slow to promote the formation of the gel directly within the syringe, and prior to injection. Nevertheless, it was necessary to find the best possible compromise with the crosslinking 
rate, to prevent hydroxyapatite precipitation.

The internal gelation of the low methoxyl pectin used in this work occurred within 9 and 50 min after preparation, depending upon the concentration of the reagents (Table 1).

A slow gelling kinetic is needed for the loading of cells, drugs or other bioactive molecules, due to the technical time required for the immobilization process. Therefore, the hydrogels prepared in this work appears suitable for the immobilization technology. Later increase of the storage modulus was related to internal strengthening of the gel network by intermolecular forces [23], until reaching a stable gel network within the first three hours of the gel formation process. This result accomplished the desirable gelling kinetics, avoiding the use of external stimuli (temperature, $\mathrm{pH}$, photoinitiators) and preventing hydroxyapatite precipitation, providing a tight control over the final morphology and properties of the gel. The gelling time appeared mostly dependent upon the concentrations of hydroxyapatite, rather than the concentration of pectin solutions (Table 3).

The rheological tests discussed in this work give useful information for the application of these gels as injectable biomaterials. Our preliminary data already bear clear evidence of the progressive overall aging of the gel structure although a full rheological characterization is needed to understand such a complex aging behaviour $[49,50]$. However, our data can support the pectin gelling mechanism described by Braccini et al, as a cooperative two-stage process involving an initial dimerization followed by subsequent aggregation of the dimers [23].

Among the rheological parameters evaluated in this study, the storage modulus $\left(\mathrm{G}^{\prime}\right)$ provides quantitative information on the ability of the hydrogel to resist to deformation that can occur during injection or after implantation, by the neighbouring tissues. The complex viscosity instead can be related to the spreading behaviour of the hydrogel once 
injected and therefore, to its ability to remain in the desired site rather than to disperse in the physiological milieu.

The rheological properties, as well as the injection forces required to extrude the pectinhydroxyapatite biocomposites, were strictly correlated to pectin concentrations (Figures 4-6) and resulted similar to the properties of injectable hydrogels commercially available as soft tissue fillers or to the properties of hydrogels proposed in the research for cell immobilization [29-34, 51]. Furthermore, the increase of the amount of the inorganic phase improved the rheological properties of the biocomposite (Figure 5).

Thicker gels, i.e. gels with higher storage modulus and complex viscosity, that required higher extrusion force, may be felt stiffer or lumpier when injected and may potentially induce trauma to the tissue: post-injection pain, inflammation, edema and erythema. Softer gels, instead, could minimize the pain when injected but are unsuitable for load bearing tissues.

The injectability of the produced biocomposites resulted in accordance with other injectable systems found in the literature $[52,53]$. The injection of pectin biocomposites through the $30 \mathrm{G}$ needle appeared difficult for the softer formulations and not possible for those formulations with highest amounts of pectin and hydroxyapatite. As described in the literature [54], the injection force should be below $50 \mathrm{~N}$ for feasible injections and to minimize the pain during injection. Accordingly, $25 \mathrm{G}$ needle seems the most appropriate needle to be used to inject PectHA biocomposites.

When designing injectable biomaterials, the reversibility of the gelling process is an interesting clue to demonstrate the possibility of controlling the degradation or dissolution of the hydrogels after the injection.

As previous works demonstrated that the polymeric network of pectin gels is easily disrupted by competing monovalent ions [21], a saline solution $(0.9 \% \mathrm{NaCl})$ was chosen as dissolution medium. The obtained results supported the hypothesis that the gel network 
could be easily dissolved due to the competitive ion binding between sodium and calcium ions, and to the consequent disruption of the egg-box structure of the gel. This result is particularly interesting to externally modulate the release of drugs or cells possibly immobilized or to dissolve and remove the gel in the case of adverse reactions.

According to the results of the indirect cytocompatibility assay, $\mathrm{P} 3 \mathrm{H} 05$ formulation was chosen to perform a preliminary immobilization study. The aim of this characterization was to prove that the immobilization procedure was not harmful for a model cell line (L929 fibroblasts) and that the gel network was sufficiently permeable to oxygen and nutrients, to support the viability of the entrapped cells. A slight decrease of cell viability was observed in the first hour after immobilization (Figure 10), then it was maintained almost constant until the end of the experiment (24h), indicating that pectinhydroxyapatite hydrogels provide an adequate environment for the cells.

On the basis of the performed characterization, the described pectin-hydroxyapatite biocomposites show interesting features that can be further exploited with minimally invasive approaches for tissue engineering and regenerative medicine.

\section{Conclusions}

Pectin-hydroxyapatite hydrogels produced by internal gelation were developed with the purpose of designing injectable biocomposites for tissue regeneration. The biocomposites were optimized in terms of $\mathrm{pH}$, gelation kinetics and rheological properties. Varying the concentrations of the reagents (pectin and hydroxyapatite), tunable systems were obtained.

Due to their rheological and texture characteristics, pectin-hydroxyapatite hydrogels were extruded from syringes with small needle sizes, and therefore appeared 
appropriate for the development of minimally-invasive procedures. The indirect cytocompatibility assay and the immobilization test primarily confirmed the suitability of these biocomposites to support the immobilization of cells and therefore to develop injectable systems for tissue regeneration.

\section{Acknowledgements}

This work was partially supported by grants from Fondazione Cassa di Risparmio di Trento e Rovereto, SG2329/2011-2011/0206 and from the CARIPLO Foundation, Italy (Project No. 2012-0842).

The authors wish to acknowledge Herbstreith \& Fox (Neuenburg, Germany) and Eurocoating (Pergine, Trento, Italy) for kindly providing, respectively, the LM pectin and hydroxyapatite powders used in this work. The authors are also grateful to Mauro Bonvini for technical support.

\section{References}

[1] S. Ramakrishna, J. Mayer, E. Wintermantel, K.W. Leong, Biomedical applications of polymer-composite materials: a review, Composites Sci. Technol. 61 (2001) 11891224.

[2] A.K. Mohanty, M. Misra, G. Hinrichsen, Biofibres, biodegradable polymers and biocomposites: An overview, Macromol. Mater. Eng. 276-277 (2000) 1-24.

[3] B.R. Lawn, J.J. Lee, H. Chai, Teeth: Among Nature's Most Durable Biocomposites, Annu. Rev. Mater. Res. 40 (2010) 55-75. 
[4] K.A. Athanasiou, C.F. Zhu, D.R. Lanctot, C.M. Agrawal, X. Wang, Fundamentals of biomechanics in tissue engineering of bone, Tissue Eng. 6 (2000) 361-381.

[5] J.J. Marler, A. Guha, J. Rowley, R. Koka, D. Mooney, J. Upton, J.P. Vacanti, Softtissue augmentation with injectable alginate and syngeneic fibroblasts, Plast. Reconstr. Surg. 105 (2000) 2049-2058.

[6] D. Macaya, M. Spector, Injectable hydrogel materials for spinal cord regeneration: a review, Biomed. Mater. 7 (2012) 012001.

[7] A.A. Amini, L.S. Nair, Injectable hydrogels for bone and cartilage repair, Biomed. Mater. 7 (2012) 024105.

[8] C.L. Salgado, E.M.S. Sanchez, C.A.C. Zavaglia, A.B. Almeida, P.L. Granja, Injectable Biodegradable Polycaprolactone-Sebacic Acid Gels for Bone Tissue Engineering, Tissue Eng. A 18 (2012) 137-146.

[9] S.J. Bidarra, C.C. Barrias, K.B. Fonseca, M.A. Barbosa, R.A. Soares, P.L. Granja, Injectable in situ crosslinkable RGD-modified alginate matrix for endothelial cells delivery, Biomaterials 32 (2011) 7897-7904.

[10] F. Munarin, P. Petrini, S. Bozzini, M.C. Tanzi, New perspectives in cell delivery systems for tissue regeneration: naturally - derived injectable hydrogels, J. Appl. Biomater. Funct. Mater. 10 (2012) 67-81.

[11] L.D. Grunebaum, I.B. Allemann, S. Dayan, S. Mandy, L. Baumann, The risk of alar necrosis associated with dermal filler injection, Dermatol. Surg. 35 (2009) 1635-1640.

[12] U.S. Zimmermann, T.J. Clerici, The histological aspects of fillers complications, Semin. Cutan. Med. Surg. 23 (2004) 241-250.

[13] F. Munarin, S.S. Guerreiro, M.A. Grellier, M.C Tanzi, M.A. Barbosa, P. Petrini, P.L. Granja, Pectin-based injectable biomaterials for bone tissue engineering, Biomacromolecules 12 (2011) 568-577. 
[14] F. Munarin, P. Petrini, S. Farè, M.C. Tanzi, Structural properties of polysaccharidebased microcapsules for soft tissue regeneration, J. Mater. Sci. Mater. Med. 21 (2010) $365-375$.

[15] F. Munarin, P. Petrini, M.C. Tanzi, M.A. Barbosa, P.L. Granja, Biofunctional chemically modified pectin for cell delivery, Soft Matter 8 (2012) 4731-4739.

[16] H.R. Moreira, F. Munarin, R. Gentilini, L. Visai, P.L. Granja, M.C. Tanzi, P. Petrini, Injectable pectin hydrogels produced by internal gelation: $\mathrm{pH}$ dependence of gelling and rheological properties, Carbohydr. Polym. in press, DOI: 10.1016/j.carbpol.2013.12.057.

[17] H.E. Kokkonen, J.M. Ilvesaro, M. Morra, H.A. Schols, J. Tuukkanen, Effect of Modified Pectin Molecules on the Growth of Bone Cells, Biomacromolecules 8 (2007) $509-515$.

[18] C.M.G.C. Renard, J. Thibault, Degradation of pectins in alkaline conditions: kinetics of demethylation, Carbohydr. Res. 286 (1996) 139-150.

[19] Y. Fang, S. Al-Assaf, G.O. Phillips, K. Nishinari, T. Funami, P.A. Williams, Binding behavior of calcium to polyuronates: Comparison of pectin with alginate, Carbohydr. Polym 72 (2008) 334-341.

[20] I. Fraeye, I. Colle, E. Vandevenne, T. Duvetter, S. Van Buggenhout, P. Moldenaers, A. Van Loey, M. Hendrickx, Influence of pectin structure on texture of pectincalcium gels, Innov. Food Sci. Emerg. Technol. 11 (2010) 401-409.

[21] F. Munarin, M.C. Tanzi, P. Petrini, Advances in biomedical applications of pectin gels, Int. J. Biol. Macromol. 51 (2012) 681-689.

[22] I. Braccini, R.P. Grasso, S. Pérez, Conformational and configurational features of acidic polysaccharides and their interactions with calcium ions: a molecular modeling investigation, Carbohydr. Res. 317 (1999) 119-130. 
[23] I. Braccini, S. Pérez, Molecular basis of $\mathrm{C}(2+)$-induced gelation in alginates and pectins: the egg-box model revisited, Biomacromolecules 2 (2001) 1089-1096.

[24] F. Munarin, S. Bozzini, L. Visai, M.C. Tanzi, P. Petrini, Sterilization treatments on polysaccharides: effects and side effects on pectin, Food Hydrocoll. 31 (2013) 74-84.

[25] P. Petrini, M.C. Tanzi, L. Giuliano, F. Munarin, P. Robotti, G. Bianchi, Composite material comprising pectin and calcium phosphate and method for its realization, International patent n. WO/2012/007917, 2012.

[26] L. Yoo, V. Gupta, C. Lee, P. Kavehpore, J. Demer, Viscoelastic properties of bovine orbital connective tissue and fat: constitutive models, Biomech. Model. Mechanobiol. $10(2011) 901-914$.

[27] P.N. Patel, C.K. Smith, C.W.J. Patrick, Rheological and recovery properties of poly(ethylene glycol) diacrylate hydrogels and human adipose tissue, J. Biomed. Mater. Res. A 73A (2005) 313-319.

[28] M. Hrapko, J.A.W. van Dommelen, G.W.M. Peters, J.S.H.M. Wismans, The mechanical behaviour of brain tissue: large strain response and constitutive modelling, Biorheology 43 (2006) 623-636.

[29] B. Wang, Y. Cao, L. Yang, Y. Wang, Rheological properties of PLGA-PEG-PLGA copolymers for ophthalmic injection, J. Appl. Polym. Sci. 125 (2012) 370-375.

[30] J. Kablik, G.D. Monheit, L. Yu, G. Chang, J. Gershkovich, Comparative physical properties of hyaluronic acid dermal fillers., J. Dermatol. Surg. 35 (2009) 302-312.

[31] S. Santoro, L. Russo, V. Argenzio, A. Borzacchiello, , J. Appl. Biomater. Biomech. $2(2011) 127-136$.

[32] S.J. Falcone, R.A. Berg, Crosslinked hyaluronic acid dermal fillers: a comparison of rheological properties, J. Biomed. Mater. Res. A Rheological properties of crosslinked hyaluronic acid dermal fillers 87A (2008) 264-271. 
[33] H. Sundaram, B. Voigts, K. Beer, M. Meland, Comparison of the rheological properties of viscosity and elasticity in two categories of soft tissue fillers: calcium hydroxylapatite and hyaluronic acid, Dermatol. Surg. 36 (2010) 1859-1865.

[34] B.A. Aguado, W. Mulyasasmita, J. Su, J.; K.J. Lampe, S.C. Heilshorn, Improving viability of stem cells during syringe needle flow through the design of hydrogel cell carriers, Tissue Eng. A. 18 (2012) 806-815.

[35] H.H. Winter, Gel Point, Encyclopedia of Polymer Science and Technology, John Wiley \& Sons, Inc. 2002.

[36] L. Bohlin, A theory of flow as a cooperative phenomenon, J. Colloid Interface Sci. $74(1980) 423-434$.

[37] H.H. Winter, F. Chambon, Analysis of Linear Viscoelasticity of a Crosslinking Polymer at the Gel Point, J. Rheol., 30 (1986) 367-382.

[38] P.G. de Gennes, Scaling Concepts in Polymer Physics; Cornell University Press, Ithaca, USA, 1979.

[39] T. Mita, L. Bohlin, Shear stress relaxation of chemically modified gluten, Cereal Chem. 60 (1983) 60-93.

[40] D. Gabriele, B. Cindio, P. D'Antona, A weak gel model for foods, Rheol. Act. 40 (2001) 120-127.

[41] W. Zhao, X. Jin, Y. Cong, Y. Liu, J. Fu, Degradable natural polymer hydrogels for articular cartilage tissue engineering, J. Chem. Technol. Biotechnol. 88 (2013) 327339.

[42] F. Khan, S.R. Ahmad, Polysaccharides and their derivatives for versatile tissue engineering application, Macromol. Biosci. 13 (2013) 395-421.

[43] M. Swetha, K. Sahithi, A. Moorthi, N. Srinivasan, K. Ramasamy, N. Selvamurugan, Biocomposites containing natural polymers and hydroxyapatite for bone tissue engineering, Int. J. Biol. Macromol. 47 (2010) 47, 1-4. 
[44] G. Turco, E. Marsich, F. Bellomo, S. Semeraro, I. Donati, F. Brun, M. Grandolfo, A. Accardo, S. Paoletti, Alginate/Hydroxyapatite Biocomposite For Bone Ingrowth: A Trabecular Structure With High And Isotropic Connectivity, Biomacromolecules, 10 (2009) 1575-1583.

[45] C. Korach, G. Halada, H. Mubarez, Effects of Processing Conditions on ChitosanHydroxyapatite Biocomposite Mechanical Properties. Springer, New York, USA 2011, p. 125-130.

[46] A.S. Asran, S. Henning, G.H. Michler, Polyvinyl alcohol-collagen-hydroxyapatite biocomposite nanofibrous scaffold: Mimicking the key features of natural bone at the nanoscale level, Polymer 51 (2010) 868-876.

[47] F.L. De Paula, I.C. Barreto, M. H. Rocha-Leao, R. Borojevic, A.M. Rossi, F.P. Rosa, M. Farina, droxyapatite-alginatebiocomposite promotes bone mineralization in different length scales in vivo, Front. Mater. Sci. China 3 (2009) 145-153.

[48] F. Brun, G. Turco, A. Accardo, S. Paoletti, Automated quantitative characterization of alginate/hydroxyapatite bone tissue engineering scaffolds by means of micro-CT image analysis, J. Mater. Sci. Mater. Med. 22 (2011) 2617-2629.

[49] H.H. Winter, Transient Networks, Progress Colloid Polym. Sci. 75 (1987) 104-110.

[50] E. Secchi, T. Roversi, S. Buzzaccaro, L. Piazza, R. Piazza, Biopolymer gels with physical cross-links: gelation kinetics, aging, heterogeneous dynamics, and macroscopic mechanical properties, Soft Matter 9 (2013) 3931-3944.

[51] A. Fattahi, P. Petrini, F. Munarin, Y. Shokoohinia, M.A. Golozar, J. Varshosaz, M.C. Tanzi, Polysaccharides Derived from Tragacanth as Biocompatible Polymers and Gels, J. Appl. Polym. Sci. 129 (2013) 2092-2102.

[52] W. Rungseevijitprapa, R. Bodmeier, Injectability of biodegradable in situ forming microparticle systems (ISM), European J. Pharm. Sci. 36 (2009) 524-531. 
[53] S.M. Oliveira, I.F. Almeida, P.C. Costa, C.C. Barrias, M.R.P. Ferreira, M.F. Bahia, M.A. Barbosa, Characterization of Polymeric Solutions as Injectable Vehicles for Hydroxyapatite Microspheres, AAPS Pharm. Sci. Tech. 11 (2010) 852-858.

[54] W. Rungseevijitprapa, R. Bodmeier, Injectability of biodegradable in situ forming microparticle systems (ISM), European J. Pharm. Sci. 36 (2009) 524-531. 